\title{
CARACTERIZACIÓN FÍSICA Y QUÍMICA DE VINOS TINTOS PRODUCIDOS EN QUERÉTARO
}

\section{PHYSICAL AND CHEMICAL CHARACTERIZATION OF RED WINES PRODUCED IN QUERÉTARO}

\author{
Mario A. de la Cruz-de Aquino', Ramón A.

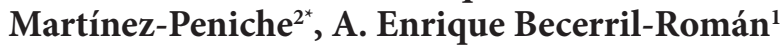 \\ y Ma. del Socorro Chávaro-Ortiz ${ }^{2}$
}

\begin{abstract}
${ }^{1}$ Postgrado en Recursos Genéticos y Productividad-Fruticultura, Colegio de Postgraduados - Campus Montecillo. Km 36.5 Carr. México-Texcoco. 56230, Montecillo, Texcoco, Estado de México. ${ }^{2}$ Departamento de Investigación y Posgrado en Alimentos, Universidad Autónoma de Querétaro, Facultad de Química. 76000, Querétaro, Qro. México.
\end{abstract}

^Autor para correspondencia (alvar@uaq.mx)

\section{RESUMEN}

La calidad del vino está determinada por el cultivar, las prácticas de cultivo, el suelo, el clima y el proceso de elaboración del vino. En el Estado de Querétaro, México hay diversas regiones vitícolas que varían en clima y tipo de suelo, donde la superficie plantada con cultivares de calidad para la elaboración de vino tinto se ha incrementado en los últimos años. Para determinar la calidad de vinos tintos obtenidos con uvas 'Cabernet Sauvignon' y 'Merlot' establecidos en tres viñedos en diferentes regiones de Querétaro se efectuaron microvinificaciones tradicionales, se dio seguimiento a la fermentación, y los vinos fueron clarificados y analizados. Los vinos de El Rosario mostraron menor grado alcohólico (8.5 GL) que los de El Barreno y que los de Viñedos Azteca (11.0 GL y 11.8 GL, respectivamente), así como mayor acidez (ATT = 7.9 vs. 6.5 y $7.7 \mathrm{~g} \mathrm{~L}^{-1}$ ác. tartárico), mientras que los vinos de Viñedos Azteca mostraron los máximos valores de intensidad colorante $(\mathrm{IC}=9.9)$ y antocianinas totales $\left(\mathrm{AT}=893.6 \mathrm{mg} \mathrm{L}^{-1}\right)$. En cuanto a los cultivares, los vinos de 'Merlot' obtuvieron el mayor grado alcohólico $\left(11.7^{\circ} \mathrm{GL}\right)$, menor ATT $\left(6.9 \mathrm{~g} \mathrm{~L}^{-1}\right)$ y mayor AT $\left(751.1 \mathrm{mg} \mathrm{L}^{-1}\right)$ que los de 'Cabernet Sauvignon' (9.1 ${ }^{\circ} \mathrm{GL}, 9.1 \mathrm{~g} \mathrm{~L}^{-1}$ y $617.2 \mathrm{mg} \mathrm{L}^{-1}$, respectivamente). Los niveles de $\mathrm{SO}_{2}$ y acidez volátil en todos los vinos se encontraron por debajo de los límites legales. Se detectaron interacciones significativas (cultivar $\mathrm{x}$ viñedo; $\mathrm{P} \leq \mathbf{0 . 0 5}$ ) en las principales variables analizadas. De acuerdo con sus características físicas y químicas, los mejores vinos fueron los obtenidos con uva 'Merlot' producida en Ezequiel Montes.

Palabras clave: Vitis vinifera, regionalización, calidad del vino, microvinificación.

\section{SUMMARY}

Wine quality is determined by cultivar, agricultural practices, soil, climate, and winemaking technique. In Querétaro, México several viticultural regions with different climates and soils can be distinguished, and the area planted with appropriate red wine cultivars has increased in the last years. To determine the quality of the red wines obtained from 'Cabernet Sauvignon' and 'Merlot' grapes in three vineyards located in different regions of the State of Querétaro, traditional microscale winemaking was carried out, monitoring fermentation evolution. Wines were later clarified and analyzed. Wines from El Rosario showed minor alcohol content (8.5 GL) than those from El Barreno and Viñedos Azteca (11.0 GL and $11.8 \mathrm{GL}$, respectively) and higher acidity (TTA = 7.9 vs. 6.5 y $7.7 \mathrm{~g} \mathrm{~L}^{-1}$ tartaric acid), while wines from Viñedos Azteca showed the highest colorant intensity values $(\mathrm{IC}=9.9)$ and total antocyanines ( $\left.\mathrm{TA}=893.6 \mathrm{mg} \mathrm{L}^{-1}\right)$. In terms of cultivars, 'Merlot' wines had the highest alcohol content $\left(11.7^{\circ} \mathrm{GL}\right)$, the lowest TTA $\left(6.9 \mathrm{~g} \mathrm{~L}^{-1}\right)$ and a high TA (751.1 $\mathrm{mg} \mathrm{L}^{-1}$ ) compared to wines from 'Cabernet Sauvignon' ( $9.13{ }^{\circ} \mathrm{GL}, 9.1 \mathrm{~g} \mathrm{~L}^{-1}$ and $617.2 \mathrm{mg} \mathrm{L}^{-1}$, respectively). $\mathrm{SO}_{2}$ content and volatile acidity in all the wines were below the legal limits. Significant interactions (cultivar $x$ vineyard; $P \leq 0.05$ ) were found in the principle variables evaluated. According to their physical and chemical characteristics, the best wines were those obtained from 'Merlot' grape grown at Ezequiel Montes.

Index words: Vitis vinifera, regionalization, wine quality, microscale winemaking.

\section{INTRODUCCIÓN}

El vino es la bebida que resulta de la fermentación alcohólica del mosto de uvas (Vitis vinifera) (Navarre, 1998). Existen factores como el proceso de vinificación, la variedad y el método de cultivo, que marcan diferencias en los atributos de un vino (Kennedy, 2008); sin embargo, el llamado terruño (terroir), influenciado por la luminosidad, altitud, latitud, régimen pluvial, pendiente, orientación y tipo de suelo, es el que define la tipicidad de la uva y del vino (Torres et al., 2006), lo cual se expresa en el contenido de azúcar, acidez, color y aroma, entre otros (Tonietto, 2007). En general, la calidad de una vendimia está determinada por la interacción del cultivar con el suelo y el clima predominante en una región, en un año determinado (Ferrer et al., 2007).

El consumo de vino en México es de 535000 hL anuales, de los cuales más de $70 \%$ se importa y el consumo per capita de vino es de sólo $530 \mathrm{~mL}$ (OIV, 2007); los vinos nacionales se producen en diferentes zonas geográficas con características climáticas y geográficas dispares (De Blas, 1998).

En el Estado de Querétaro, México, situado en la zona centro del país, se distinguen al menos dos regiones vitícolas, una de ellas correspondiente al Valle de San Juan del Río, cuyo clima es semiseco templado y donde predominan los suelos planos, profundos con tendencia arenoarcillosa; y la otra región está situada dentro de los municipios de Tequisquiapan y Ezequiel Montes, donde existen mayores condiciones de aridez, con suelos en pendientes y más calcáreos ("tepetatosos", con una capa dura en el subsuelo).

Después de haber sufrido una severa crisis en los años 80 y 90 debida a los altos costos de producción, bajos precios de la uva, desconocimiento de la tecnología de producción y las importaciones (Martínez-Peniche, 1995), la industria vitivinícola queretana ha resurgido, con una tendencia al incremento en la superficie establecida y a la mejora de la calidad de sus uvas y sus vinos, fundamentalmente tintos, para lo cual se han establecido nuevas plantaciones con variedades finas como 'Merlot', 'Cabernet Sauvignon', 'Syrah', 'Tempranillo', etc., y en la actualidad la superficie 
establecida con viñedos es de 212 ha (AVQ, 2011). Sin embargo, debido al desconocimiento del potencial de las distintas regiones para la producción de vinos de calidad, este crecimiento no ha sido ordenado. El determinar la vocación de las regiones vitícolas para cada cultivar permitirá producir vinos típicos de calidad comercializables a precios rentables, lo cual a su vez coadyuvará al desarrollo de esta agroindustria.

Por lo anterior, el objetivo de esta investigación fue determinar la calidad física y química de vinos tintos obtenidos con uvas de dos cultivares establecidos en tres regiones del Estado de Querétaro.

\section{MATERIALES Y MÉTODOS}

\section{Sitio experimental y material biológico}

El presente trabajo se llevó a cabo en tres viñedos establecidos en distintos municipios del Estado de Querétaro, México. La localización geográfica de los viñedos se precisa en la Figura 1 y sus principales características climáticas y de ubicación se resumen en el Cuadro 1.
Los cultivares incluidos en este estudio fueron 'Cabernet Sauvignon' y 'Merlot'. Los vinos del primero poseen un aroma herbáceo y especiado pronunciado; de buena acidez y buen color; requieren de añejamiento para lograr un balance excelente (Galet, 1985). Los vinos de 'Merlot' manifiestan un color rubí intenso con tintes violáceos el cual depende de la zona de producción, presentan aromas de casis, grosella, mora u otros frutos rojos, pimiento dulce, humo, guinda, violeta además de trufas y el cuero, de sabor agradable cuando joven por no contener una alta cantidad taninos (Amerine y Roessler, 1958).

\section{Conducción del experimento}

La cosecha se llevó a cabo manualmente el 5 de agosto de 2011 para obtener $20 \mathrm{~kg}$ por cultivar en cada uno de los tres viñedos. La uva obtenida fue microvinificada por el método tradicional, con las siguientes operaciones (Ibacache, 2010): 1) Estrujado y despalillado, con una estrujadora mecánica de $120 \mathrm{~V}$ y capacidad para $2 \mathrm{t} \mathrm{h}^{-1}$; 2) Encubado, en el que el mosto fue depositado en vitroleros de capacidad de $20 \mathrm{~L}$; 3) Sulfitado, en el que se adicionó metabisulfito de potasio a una dosis de $5 \mathrm{~g} \mathrm{hL}^{-1}$ (50 ppm); 4) Fermentación alcohólica y maceración, en donde la fermentación se inició mediante

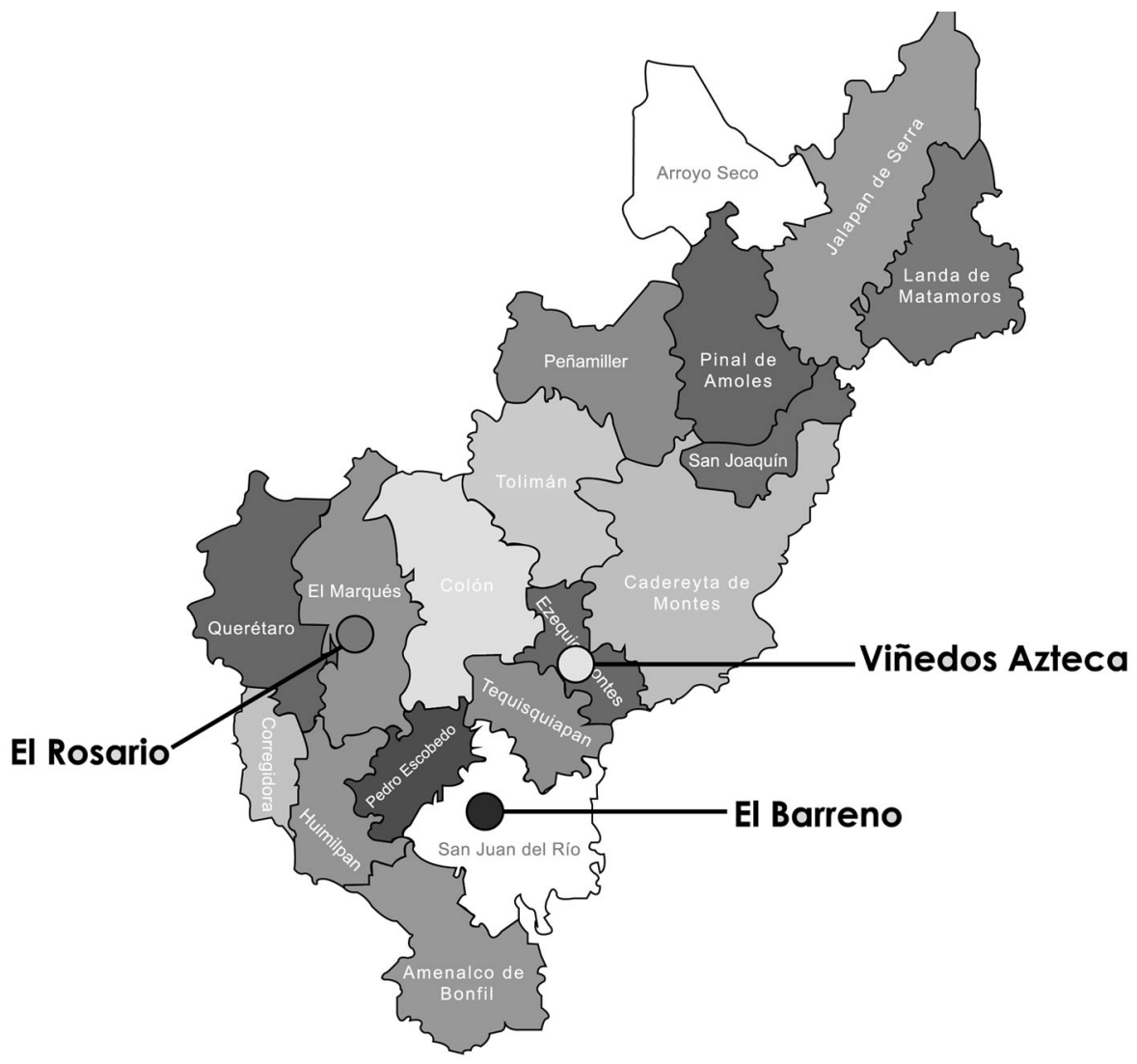

Figura 1. Localización de las viñas de procedencia de las uvas evaluadas en este estudio. 
Cuadro 1. Características de los viñedos del Estado de Querétaro evaluados en este estudio.

\begin{tabular}{lcccccccc}
\hline Viñedo & Municipio & $\begin{array}{c}\text { Latitud } \\
\text { "N" }\end{array}$ & $\begin{array}{c}\text { Longitud } \\
\text { "O”" }\end{array}$ & $\begin{array}{c}\text { Altitud } \\
(\mathrm{m})\end{array}$ & $\begin{array}{c}\text { TMA } \\
\left({ }^{\circ} \mathrm{C}\right)\end{array}$ & $\begin{array}{c}\text { PMA } \\
(\mathrm{mm})\end{array}$ & $\mathrm{UF}^{\dagger}$ & $\mathrm{UC}^{\dagger \dagger}$ \\
\hline El Rosario & El Marqués & $20^{\circ} 58^{\prime}$ & $100^{\circ} 09^{\prime}$ & 1850 & 16.7 & 485.3 & 376 & 1799.6 (III) \\
El Barreno & San Juan del Río & $20^{\circ} 23^{\prime}$ & $99^{\circ} 59^{\prime}$ & 1920 & 16.7 & 580.3 & 332 & $1732.8(\mathrm{III})$ \\
Viñedos Azteca & Ezequiel Montes & $20^{\circ} 43^{\prime}$ & $99^{\circ} 44^{\prime}$ & 1950 & 16.1 & 505.0 & 474 & $1675.0(\mathrm{III})$ \\
\hline
\end{tabular}

${ }^{\dagger}$ Calculado con el método Richardson et. al. (1974) con datos de las estaciones de Chichimequillas para El Rosario, de San Juan del Río para El Barreno y de Cadereyta para Viñedos Azteca.

${ }^{\dagger+}$ Calculado a partir de las temperaturas medias de abril a octubre del año 2010; entre paréntesis, región climática, según el método de Winkler (1980). TMA = temperatura media anual; PMA = precipitación media anual.; UF = unidades frío; UC = unidades calor.

inoculación de los mostos con Saccharomyces cerevisiae en polvo liofilizada a una concentración de $400 \mathrm{mg} \mathrm{L}^{-1}$. El tiempo de maceración para todos los vinos fue de $5 \mathrm{~d}$, durante los cuales se hicieron dos bazuqueos diarios; 5) Descube, en el que se obtuvo el vino flor por escurrido y el vino de prensa con una prensa vertical manual de aluminio. Ambos vinos fueron mezclados para continuar con la fermentación terminal, la cual se consideró terminada cuando la densidad del vino llegó a alrededor de $0.996 \mathrm{~g} \mathrm{~mL}^{-1}$; los vinos fueron entonces traspasados a recipientes de $1.8 \mathrm{~L}, \mathrm{y}$ 6) Clarificación de los vinos, que se hizo con $80 \mathrm{~mL} \mathrm{hL}^{-1}$ de clara de huevo como fuente de albúmina.

\section{Análisis físicos y químicos}

Se hicieron las siguientes determinaciones: 1) Densidad $(\delta)$ por medio de picnometría, para el mosto y para el seguimiento de la fermentación; 2) Acidez total titulable (ATT) mediante titulación (CEE, 1990); 3) pH con un potenciómetro calibrado a dos puntos; 4) Acidez volátil (AV) por el método García-Tena (García, 1976); 5) Grado alcohólico $\left({ }^{\circ} \mathrm{GL}\right)$ por destilación directa (CEE, 1990); 6) Anhídrido sulfuroso $\left(\mathrm{SO}^{2}\right)$ libre, combinado y total, por el método Rippert (CEE, 1990); 7) Características cromáticas [intensidad colorante (IC) y matiz] por el método de la OIV (2012); y 8) Antocianinas totales (AT) mediante decoloración de sulfuroso (CEE, 1990).

\section{Análisis estadísticos}

El diseño experimental fue completamente al azar con cuatro repeticiones y arreglo factorial de tratamientos, cuyos factores fueron los cultivares con dos niveles: ('Cabernet Sauvignon', y 'Merlot'), y viñedos con tres niveles (El Rosario, El Barreno y Viñedos Azteca). La unidad experimental consistió de un vitrolero de $1.8 \mathrm{~L}$. Se realizó el análisis de varianza y la prueba de medias de " $\mathrm{t}$ " de Student $(\mathrm{P} \leq 0.05)$ , con el programa estadístico STATGRAPHICS Centurion XVI.I (Castaño y Domínguez, 2010).

\section{RESULTADOS Y DISCUSIÓN}

\section{Evolución de la fermentación}

La densidad $(\delta)$ inicial de 'Cabernet Sauvignon' fue inferior en los mostos provenientes de El Rosario $\left(1.067 \mathrm{~g} \mathrm{~L}^{-1}\right)$ que en la de los otros viñedos, en los que resulta comparable (1.083 $\mathrm{g} \mathrm{L}^{-1}$ para El Barreno y $1.082 \mathrm{~g} \mathrm{~L}^{-1}$ para Viñedos Azteca). Estas $\delta$ corresponden a $154.8 \mathrm{~g} \mathrm{~L}^{-1}$ de azúcar para el Rosario, $195.3 \mathrm{~g} \mathrm{~L}^{-1}$ para el Barreno y $193.1 \mathrm{~g} \mathrm{~L}^{-1}$ para Viñedos Azteca (CEE, 1990). Aunque el desarrollo de la fermentación fue homogéneo en los mostos de los tres viñedos, el vino producido con uva de El Rosario obtiene una $\delta$ final ligeramente superior a la de los otros dos viñedos $(0.998 \mathrm{~g}$ $\mathrm{L}^{-1}$ vs. $0.994 \mathrm{~g} \mathrm{~L}^{-1}$ para el Barreno y $0.996 \mathrm{~g} \mathrm{~L}^{-1}$ para Viñedos Azteca), valores que corresponden a fermentaciones completas (Navarre, 1998) (Figura 2A).

En el caso de 'Merlot', el mosto de El Rosario nuevamente presentó la menor densidad (1.075), correspondiente a una concentración de azúcares y un grado alcohólico potencial inferiores a los de los otros dos viñedos. La densidad en los mostos de El Barreno fue comparable a la de los de Viñedos Azteca (1.098 vs. 1.100, respectivamente). Además, la fermentación de los vinos de El Rosario, se completa en sólo $2 \mathrm{~d}$, mientras que los vinos de los otros dos viñedos llegan a una $\delta$ inferior a 1.0 hasta el $4^{\circ}$ día (Figura 2B). Finalmente, el contenido inicial de azúcar en 'Merlot' en cada uno de los viñedos (174.9 $\mathrm{g} \mathrm{L}^{-1}$ en El Rosario, $234.6 \mathrm{~g} \mathrm{~L}^{-1}$ en El Barreno y $239.3 \mathrm{~g} \mathrm{~L}^{-1}$ en Viñedos Azteca) es superior que en 'Cabernet Sauvignon'.

El hecho de que los mostos de uva de los dos cultivares provenientes de El Rosario hayan obtenido una menor concentración de azúcar que la de los otros dos viñedos se debe a un retraso en la maduración de los frutos, posiblemente debido a una menor acumulación de calor, ya que la temperatura promedio para los meses de julio y agosto de 2011 fue de $20.2^{\circ} \mathrm{C}$ [ 632.4 unidades calor (UC), de acuerdo con Winkler (1980)] en ese viñedo, mientras que para El Barreno fue de $20.7^{\circ} \mathrm{C}$ (663.4 UC) y para Viñedos Azteca de $20.9^{\circ} \mathrm{C}$ (675.8 UC). 

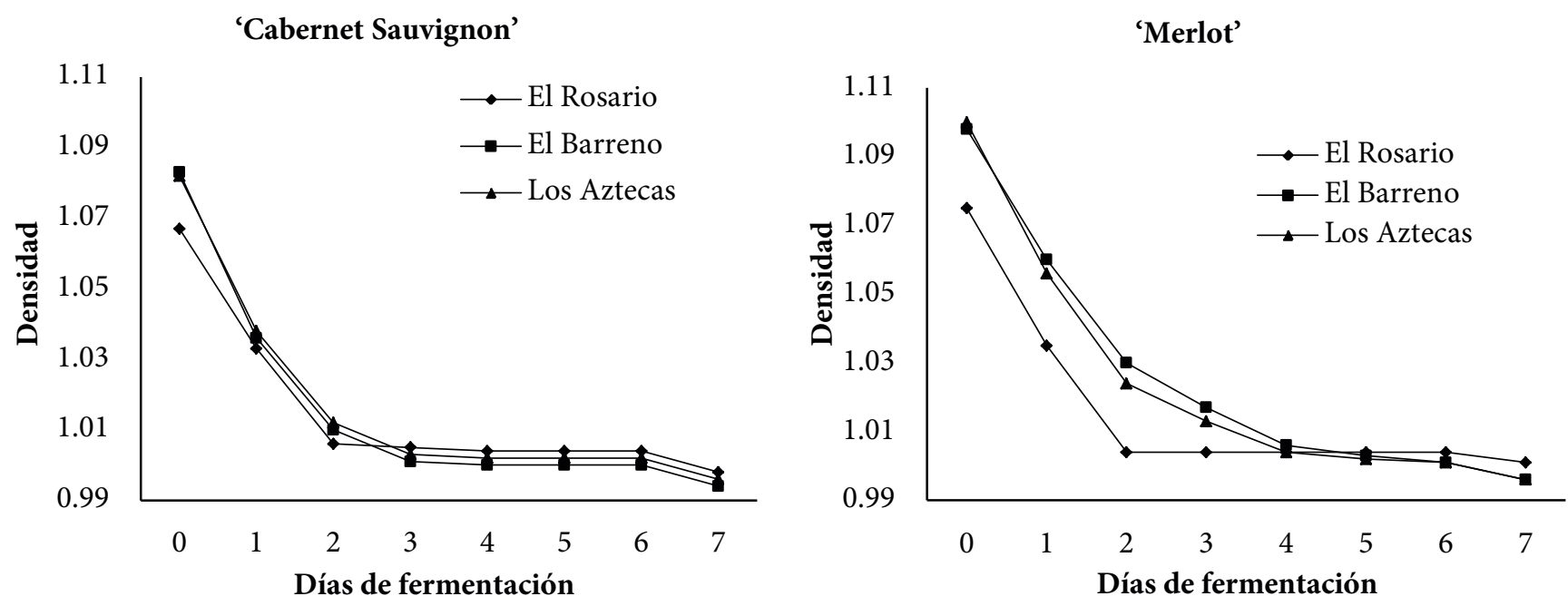

Figura 2. Evolución de la densidad durante la fermentación de los mostos de uva de dos cultivares crecidos en tres regiones del Estado de Querétaro.

\section{Caracterización física y química}

En el Cuadro 2 se advierten diferencias entre viñedos (uno de los factores de estudio) en todas las variables evaluadas. El menor grado de alcohol promedio obtenido en El Rosario $\left(8.46^{\circ} \mathrm{GL}\right)$ se debe a la menor concentración de azúcares en las uvas producidas en éste (Figura 2). Destacan los vinos de Viñedos Azteca, con el más alto grado alcohólico ( $\left.11.83^{\circ} \mathrm{GL}\right)$, seguidos de los de El Barreno (10.98 $\left.{ }^{\circ} \mathrm{GL}\right)$. El grado alcohólico real resultó inferior al potencial (9.20 ${ }^{\circ} \mathrm{GL}$ para El Rosario, $12.84^{\circ} \mathrm{GL}$ para Viñedos Azteca y $12.16^{\circ} \mathrm{GL}$ para El Barreno) (CEE, 1990). Lo anterior puede deberse a la oscilación de temperatura o a las condiciones de nutrición para el desarrollo de las levaduras durante la fermentación (Millán y Ortega, 1988).

Por otro lado, se advierte una menor acidez total titulable (ATT) en los vinos de El Barreno. Como Querétaro es una región donde se obtiene una ATT elevada, pero se requiere que para el balance en el sabor de los vinos tintos ésta sea baja, se busca la fermentación maloláctica (Navarre, 1998). Una menor ATT en el vino puede interpretarse como una característica de buena calidad. Finalmente, conforme disminuye la ATT de los vinos su pH aumenta, ya que la acidez está determinada por la concentración de ácidos orgánicos presentes (ácido málico, tartárico, cítrico, etc.) (Dequin et al., 1999).

Los vinos de Viñedos Azteca manifiestan los más altos valores de intensidad del color (IC) y antocianos totales (AT), características deseables en los vinos tintos. Lo anterior puede deberse a la mayor acumulación de azúcares en la uva. Se sabe que la IC en un vino está determinada por la concentración y tipo de antocianos, y una de las vías de su síntesis en la uva es a partir de la glucosa (Champagnol, 1984).

Con respecto a los cultivares (el segundo factor en

Cuadro 2. Promedios de grado alcohólico $\left({ }^{\circ} \mathrm{GL}\right)$, acidez total titulable (ATT, en $\mathrm{g} \mathrm{L}^{-1}$ de ácido tartárico), $\mathrm{pH}$, intensidad colorante (IC), matiz y concentración de antocianos totales (AT, en $\mathrm{mg} \mathrm{L}^{-1}$ ) en cada viñedo

\begin{tabular}{lcccccc}
\hline Viñedo & ${ }^{\circ} \mathrm{GL}^{\dagger}$ & $\mathrm{ATT}$ & $\mathrm{pH}$ & $\mathrm{IC}$ & Matiz & AT \\
\hline El Rosario & $8.46^{\mathrm{z}} \mathrm{C}$ & $7.90^{\mathrm{z}} \mathrm{a}$ & $3.35^{\mathrm{z}} \mathrm{b}$ & $7.77^{\mathrm{z}} \mathrm{b}$ & $1.38^{\mathrm{z} a}$ & $540.90{ }^{\mathrm{z}} \mathrm{b}$ \\
El Barreno & $10.98 \mathrm{~b}$ & $6.50 \mathrm{~b}$ & $3.41 \mathrm{a}$ & $9.47 \mathrm{a}$ & $1.05 \mathrm{~b}$ & $618.00 \mathrm{~b}$ \\
Viñedos Azteca & $11.83 \mathrm{a}$ & $7.70 \mathrm{a}$ & $3.30 \mathrm{c}$ & $9.87 \mathrm{a}$ & $1.07 \mathrm{~b}$ & $893.60 \mathrm{a}$ \\
Valor de F & $112.40^{* *}$ & $24.50^{* *}$ & $13.70^{* *}$ & $16.70^{\star *}$ & $16.30^{* *}$ & $22.70^{* *}$ \\
DMS & 0.51 & 0.50 & 0.05 & 0.84 & 0.14 & 116.70 \\
\hline
\end{tabular}

${ }^{2}$ Promedios con letras iguales no son estadísticamente diferentes (Student, 0.05 ); ${ }^{\dagger}$ Promedios obtenidos de ocho datos individuales. DMS $=$ diferencia mínima significativa; ${ }^{* *}$ : significativo con $\mathrm{P} \leq 0.01 . \mathrm{IC}=(\delta)$ medida en una celda de $1 \mathrm{~cm}$ de espesor. 
estudio), los vinos de 'Merlot' presentan un mayor grado alcohólico, una menor ATT y una mayor concentración de AT que los de 'Cabernet Sauvignon' (Cuadro 3). Se sabe que los vinos de 'Merlot' son bien coloreados y alcoholizados (Peñín, 1997). El contenido de antocianos totales reportados para este cultivar va de 430.5 a $730.5 \mathrm{mg} \mathrm{L}^{-1}$ (González et al., 2001).

Se advierten interacciones significativas entre el viñedo y el cultivar en prácticamente todas las variables consideradas, entre las que destacan dos de las características más deseables en los vinos tintos de calidad: grado alcohólico y AT.

En la Figura 3 se observa que en Viñedos Azteca se obtienen los vinos de 'Merlot' con el mayor grado de alcohol $\left(14.3^{\circ} \mathrm{GL}\right)$, mientras que en El Barreno el mayor valor se obtiene en 'Cabernet Sauvignon' (10.3 $\left.{ }^{\circ} \mathrm{GL}\right)$, lo que explica la interacción. Los menores valores de grado alcohólico para los dos cultivares se obtienen en El Rosario, lo que confirma el retraso en la maduración en este sitio. Además, en todos los viñedos el mayor valor de ${ }^{\circ} \mathrm{GL}$ corresponde a 'Merlot', lo que muestra un mayor potencial de este cultivar en las distintas regiones del Estado de Querétaro para producir vinos más alcoholizados.

La mayor concentración de antocianos totales se obtiene nuevamente en los vinos 'Merlot' de Viñedos Azteca (Figura 4), lo que podría deberse a la mayor cantidad de azúcares presentes en su mosto, ya que la principal ruta de síntesis de antocianos parte de la glucosa (Champagnol,

Cuadro 3. Grado alcohólico $\left({ }^{\circ} \mathrm{GL}\right)$, acidez total titulable (ATT), pH, intensidad colorante (IC), matiz y concentración de antocianos totales (AT) en vinos de dos cultivares establecidos en tres viñedos del Estado de Querétaro, y la significancia de las correspondientes interacciones

\begin{tabular}{lcccccc}
\hline Cultivar & ${ }^{\circ} \mathrm{GL}^{\dagger}$ & $\mathrm{ATT}$ & $\mathrm{pH}$ & $\mathrm{IC}$ & Matiz & $\mathrm{AT}$ \\
\hline 'C. Sauvignon' & $9.13 \mathrm{~b}$ & $7.83 \mathrm{a}$ & $3.38 \mathrm{a}$ & $9.05 \mathrm{a}$ & $1.12 \mathrm{a}$ & $617.20 \mathrm{~b}$ \\
'Merlot' & $11.72 \mathrm{a}$ & $6.90 \mathrm{~b}$ & $3.33 \mathrm{~b}$ & $9.02 \mathrm{a}$ & $1.22 \mathrm{a}$ & $751.10 \mathrm{a}$ \\
Valor de F & $187.60^{* *}$ & $25.90^{* *}$ & $7.30^{*}$ & $0.10^{\text {ns }}$ & $2.94^{\text {ns }}$ & $9.45^{\star *}$ \\
DMS & 0.40 & 0.93 & 0.04 & 0.67 & 0.11 & 92.89 \\
F de interacción & $38.10^{* *}$ & $5.60^{*}$ & $19.10^{* *}$ & $2.83^{\text {ns }}$ & $1.98^{\text {ns }}$ & $11.10^{* *}$ \\
\hline
\end{tabular}

Promedios con letras iguales no son estadísticamente diferentes (Student, 0.05 ); ${ }^{\dagger}$ Promedios obtenidos de 12 datos individuales. DMS $=$ diferencia mínima significativa; ns, ${ }^{*}, * *$ : No significancia, significativo con $\mathrm{P} \leq 0.05$, y significativo con $\mathrm{P} \leq 0.01$, respectivamente. ATT $=\mathrm{gL}^{-1}$ Ac. Tartárico; IC $=(\delta)$ medida en celda de $1 \mathrm{~cm} ; \mathrm{AT}=\mathrm{mg} \mathrm{L}^{-1}$.

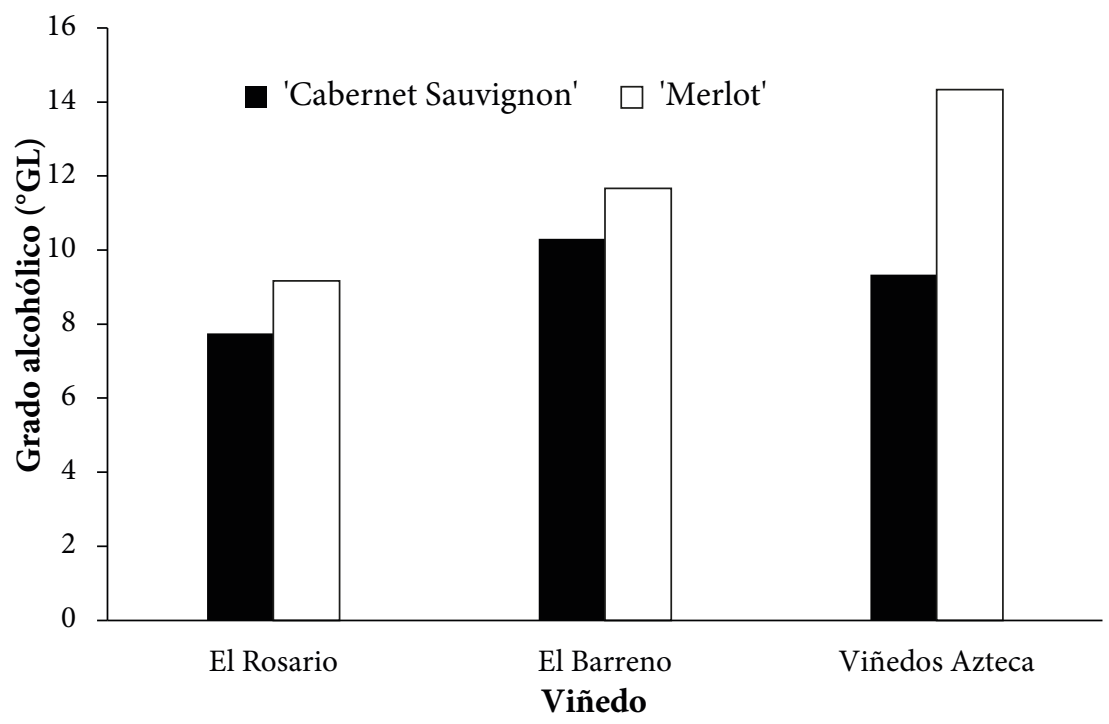

Figura 3. Grado alcohólico obtenido en vinos provenientes de uva de dos cultivares establecidos en tres viñedos del Estado de Querétaro. 
1984). Estos resultados, aunados a los obtenidos en ${ }^{\circ} \mathrm{GL}$, ponen en evidencia el alto potencial de esta región para la producción de vinos de 'Merlot'. Sin embargo, a diferencia de lo que ocurre con el grado alcohólico, los mayores valores de AT en El Barreno son los de 'Cabernet Sauvignon', lo que explica la respectiva interacción. Nuevamente los menores valores para AT en los dos cultivares se obtienen en El Rosario.

Se observan diferencias en las concentraciones de $\mathrm{SO}_{2}$ libre, $\mathrm{SO}_{2}$ combinado y acidez volátil (AV) entre viñedos (Cuadro 4). La mayor concentración de $\mathrm{SO}_{2}$ libre se obtiene en El Rosario, mientras que la de $\mathrm{SO}_{2}$ combinado se obtiene en Viñedos Azteca. Sin embargo, los valores de $\mathrm{SO}_{2}$ total son iguales para todos los vinos debido a que se aplicó una dosis única en los mostos, y estos valores no rebasan los límites legales (160 $\mathrm{mg} \mathrm{L}^{-1}$ para vinos tintos con menos de $5 \mathrm{~g} \mathrm{~L}^{-1}$ de azúcar) (Navarre, 1998). Las diferencias en $\mathrm{SO}_{2}$ libre y $\mathrm{SO}_{2}$ combinado en los dos viñedos podrían deberse a las diferencias en azúcar (Figura 2), ya que se sabe que a mayor nivel de azúcar en el mosto, el $\mathrm{SO}_{2}$ se combina más (Navarre, 1998).

Los valores de AV obtenidos (entre 0.32 y $0.37 \mathrm{~g} \mathrm{~L}^{-1}$ ác. acético, correspondientes a 0.26 y $-0.30 \mathrm{~g} \mathrm{~L}^{-1}$ ác. sulfúrico), se encuentran por debajo de los límites legales de la Unión Europea (0.98 $\mathrm{g} \mathrm{L}^{-1}$ ác. sulfúrico) (CEE, 1990) y la OIV (2012) ( $1 \mathrm{~g} \mathrm{~L}^{-1}$ de ác. acético), pues con valores mayores el vino se considera "picado" y no puede ser comercializado.
La AV es la suma de los ácidos de la serie acética; el ácido. acético en los vinos proviene de la fermentación acética por bacterias del género Acetobacter, en menor proporción, de la fermentación alcohólica por levaduras (Navarre, 1998).

Se advierten niveles casi idénticos de $\mathrm{SO}_{2}$ libre, combinado y total para los dos cultivares (Cuadro 5), pero hay una mayor AV en los vinos de 'Merlot' ( $0.38 \mathrm{~g} \mathrm{~L}^{-1}$ ác. acético $v s$. $0.32 \mathrm{~g} \mathrm{~L}^{-1}$ ác. acético en 'Cabernet Sauvignon'). No obstante, en todos los casos los valores obtenidos se encuentran muy por debajo de los límites legales.

\section{CONCLUSIONES}

Los mejores vinos obtenidos en este estudio, de acuerdo con sus características químicas, son los de 'Merlot' provenientes de Ezequiel Montes porque presentan el mayor grado alcohólico y concentración de antocianos totales; los vinos de 'Cabernet Sauvignon' procedentes de este sitio resultaron sobresalientes y comparables a los de San Juan del Río. 'Merlot' obtuvo mayor grado alcohólico que 'Cabernet Sauvignon' en las tres regiones. Es recomendable la realización de análisis sensoriales de los vinos obtenidos para ampliar la caracterización de los vinos del Estado de Querétaro.

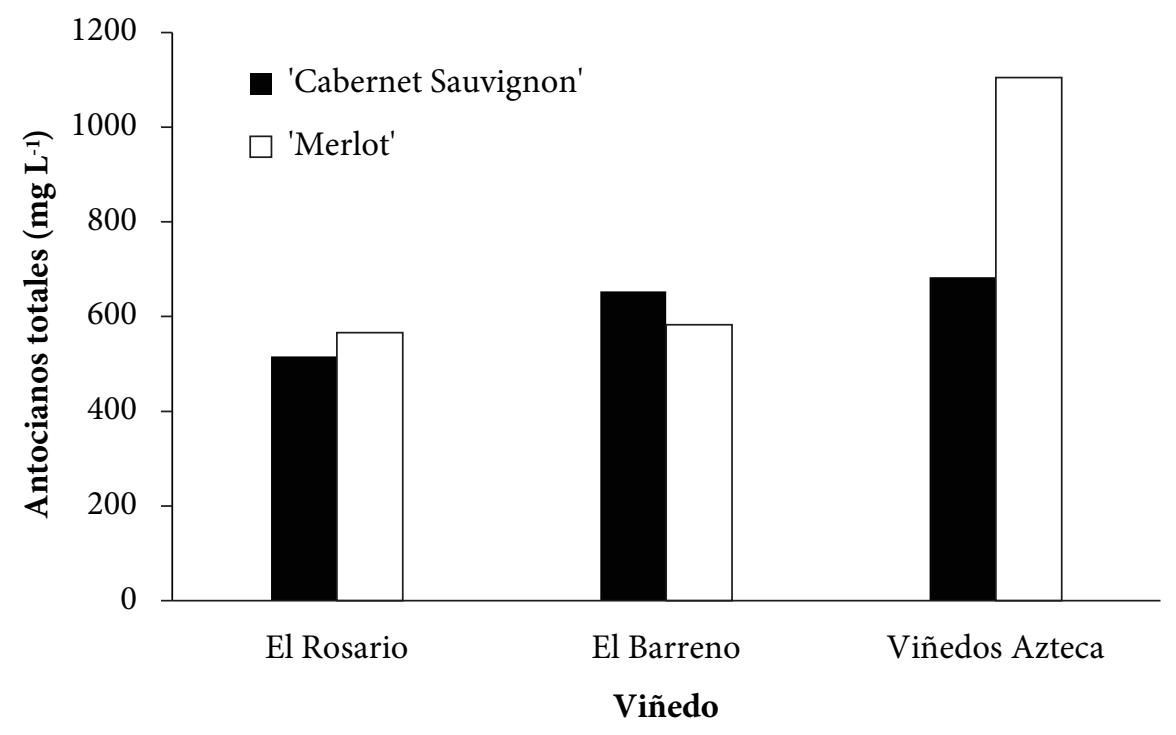

Figura 4. Concentración de antocianos totales obtenida en vinos provenientes de uva de dos cultivares establecidos en tres viñedos del Estado de Querétaro. 
Cuadro 4. Concentración de anhídrido sulfuroso $\left(\mathrm{SO}_{2}\right)$ y acidez volátil en vinos elaborados con dos cultivares, en tres viñedos del Estado de Querétaro.

\begin{tabular}{lcccc}
\hline Ubicación & ${ }^{\top} \mathrm{SO}_{2}$ libre & $\mathrm{SO}_{2}$ combinado & $\mathrm{SO}_{2}$ total & Acidez volátil \\
\hline El Rosario & $63.46 \mathrm{a}$ & $85.65 \mathrm{~b}$ & $149.13 \mathrm{a}$ & $0.35 \mathrm{a}$ \\
El Barreno & $58.59 \mathrm{~b}$ & $88.40 \mathrm{~b}$ & $147.01 \mathrm{a}$ & $0.32 \mathrm{~b}$ \\
Viñedos Azteca & $56.12 \mathrm{c}$ & $93.02 \mathrm{a}$ & $149.15 \mathrm{a}$ & $0.37 \mathrm{a}$ \\
Valor de F & $31.80^{* *}$ & $8.60^{* *}$ & $10.80 \mathrm{~ns}$ & $6.40^{* *}$ \\
DMS & 2.02 & 3.81 & 2.92 & 0.03 \\
\hline
\end{tabular}

Promedios con letras iguales no son estadísticamente diferentes (Student, 0.05 ); ${ }^{\dagger}$ Promedios obtenidos de ocho datos individuales. DMS = diferencia mínima significativa; $\mathrm{ns},{ }^{* *}$ : No significancia y significativo con $\mathrm{P} \leq 0.01$, respectivamente

Cuadro 5. Concentración de anhídrido sulfuroso $\left(\mathrm{SO}_{2}\right)$ y acidez volátil en vinos elaborados con mostos provenientes de tres viñedos del Estado de Querétaro, en función del cultivar e interacciones.

\begin{tabular}{lcccc}
\hline Cultivar & ${ }^{+} \mathrm{SO}_{2}$ libre & $\mathrm{SO}_{2}$ combinado & $\mathrm{SO}_{2}$ total & Acidez volátil \\
\hline 'Cabernet Sauvignon' & $58.76 \mathrm{a}$ & $89.30 \mathrm{a}$ & $148.07 \mathrm{a}$ & $0.32 \mathrm{~b}$ \\
'Merlot' & $60.02 \mathrm{a}$ & $88.74 \mathrm{a}$ & $148.79 \mathrm{a}$ & $0.38 \mathrm{a}$ \\
Valor de F & $2.80^{\mathrm{ns}}$ & $0.20^{\mathrm{ns}}$ & $0.40^{\mathrm{ns}}$ & $24.90^{* *}$ \\
DMS & 1.61 & 3.03 & 2.33 & 0.03 \\
Valor de F de la interacción & $5.20^{*}$ & $0.90^{\mathrm{ns}}$ & $0.30^{\mathrm{ns}}$ & $9.50^{* *}$ \\
\hline
\end{tabular}

Promedios con letras iguales no son estadísticamente diferentes (Student, 0.05); ${ }^{\dagger}$ Promedios obtenidos de 12 datos individuales. DMS = diferencia mínima significativa; $\mathrm{ns,}{ }^{*}{ }^{* *}$ : No significancia, significativo con $\mathrm{P} \leq 0.05 \mathrm{y}$ significativo con $\mathrm{P} \leq 0.01$, respectivamente.

\section{AGRADECIMIENTOS}

Al Arq. Antonino Sierra, propietario de la Finca El Rosario, al Ing. Alejandro Zendejas, propietario del Rancho El Barreno, al Ing. Jorge Ferreira propietario de Viñedos Azteca y al Ing. Alberto Rodríguez propietario de la CIA. Vinícola San Patricio S.A. de C.V., por las facilidades prestadas para la realización de este trabajo.

\section{BIBLIOGRAFÍA}

Amerin M A, E Roessler (1958) Methods of determining field maturity of grapes. Amer. J. Enol. Viticult. 9:37-40.

AVQ,. Asociación de Vitivinicultores de Querétaro (2011) Estudio de impacto productivo de un viñedo. Ed. Asociación de Vitivinicultores de Querétaro. Querétaro, México. $111 \mathrm{p}$.

Castaño T E, D J Domínguez (2010) Diseño de Experimentos: Estrategias y Análisis en Ciencia y Tecnología. Ed. Universidad Autónoma de Querétaro. Querétaro, México. 418 p.

CEE, Comunidad Económica Europea (1990) Analyses des Mouts et des Vins. Office des Publications Officielles des Communautés Européennes. Paris, France. 192 p.

Champagnol F (1984) Eléments de Physiologie de la Vigne et de la Viticulture Générale. Ed. DEHAN, Montpellier. 351 p.

De Blas J J (1998) El Vino y la Mesa. Ed. Continental. México. 150 p.

Dequin S, E Baptiste, P Barre (1999) Acidification of grape must by Saccharomyces cerevisiae. Wine yeast strains genetically engineered to produce lactic acid. Amer. J. Enol. Viticult. 50:45-50.

Ferrer M, R Peducchi, M Michalazzo, G González, A Carbonneau (2007) Delimitación y descripción de regiones vitícolas del Uruguay en base al método de clasificación climática multicriterio utilizando índices bioclimáticos adaptados a las condiciones del cultivo. Agrociencia 11: 47-56.
Galet P (1985) Précis d’Ampélographie Pratique. 5éme ed. Imprimerie DEHAN. Montpellier, France. 256 p.

García B J (1976) Metodología de Análisis de Vinos y Derivados. Ed Sepsa. Vilafranca del Penedés. España. 92 p.

González N G, C Gómez, L Barreiro (2001) Anthocyanic composition of Tannat, Cabernet Sauvignon and Merlot young wines from Uruguay. J. Wine Res. 12: 125-133.

Ibacache A (2010) Producción de vino y su relación con variables climáticas. Vendimia 78:6 10.

Kennedy J A (2008) Grape and wine phenolics: observations and recent findings. Ciencia e Investig. Agraria 35: 77-90.

Martínez-Peniche R A (1995) La industria vitivinícola de Querétaro en crisis. NTHE 13(II): 24-27.

Millan C, J M Ortega (1988) Production of ethanol, acetaldehyde, and acetic acid in wine by various yeast races: role of alcohol and aldehyde dehydrogenase. Amer. J. Enol. Viticult. 39:107-112.

Navarre J (1998) Loenologie. Ed. Lavoisier. París, Francia. 354 p.

OIV, Organisation Internationale de la Vigne et du Vin (2007) Situation et Statistiques du Secteur Vitivinicole Mondial. Ed. OIV. Paris, France. $69 \mathrm{p}$.

OIV, Organisation Internationale de la Vigne et du Vin (2012) Recuei des Méthodes Internationales d'Analyse des Vins et des Mouts. Vol.1. Ed. OIV. Paris, France. 478 p.

Peñín J (1997) Cepas del Mundo. Ed. Pi and Erre. Madrid, España. 111 p.

Richardson E A, S D Seeley, D R Walker (1974) A model for estimating the completion of rest for 'Redhaven' and 'Elberta' peach trees. HortScience 9: 331-332.

Tonietto J (2007) Evaluación climática global de las zonas vitícolas. Rev Enología. 4:24-35.

Torres M F, P C García, F A Pombo, A R Cicero (2006) Índice radiotérmico para el cultivo de la vid. Rev. Fac. Ciencias Agrarias Universidad Nal. Cuyo 38: 19-23.

Winkler A J (1980) Viticultura General. 6a ed. CECSA. D. F., México. 792 p. 